\title{
Conservative Treatment of Hemolytic Complication Following Coil Embolization in Two Adult Cases of Patent Ductus Arteriosus
}

\author{
Kazuhiko Eda, MD; Sadanori Ohtsuka, MD; Yoshihiro Seo, MD; \\ Satsuki Yamada, MD; Miki Ishiyama, MD; Tomoyuki Miyamoto, MD*; \\ Hitoshi Horigome, MD*; Iwao Yamaguchi, MD
}

\begin{abstract}
Two adult cases of relatively large patent ductus arteriosus (PDA) were treated by coil embolization, but were complicated by hemolysis that was successfully managed by medical treatment. Case 1 was a 67-year-old woman and Case 2 was a 71-year-old woman with a PDA of minimal diameter of $5.3 \mathrm{~mm}$ and $5.5 \mathrm{~mm}$, respectively. The approach was via the pulmonary artery and 2 coils were delivered simultaneously into the ductus, known as the 'kissing coil technique'. Although immediately after the procedure only a small residual shunt was revealed by aortogram, hemolysis occurred for several hours after the procedure in both cases. A hemolytic complication usually needs additional coil embolization or surgical treatment, but in these 2 cases it was successfully treated by haptoglobin infusion to prevent nephropathy and by antiplasmin infusion to promote thrombus formation. Hemolytic complications of coil embolization of PDA can managed by medication when the residual shunt is minimal and the degree of hemolysis is mild. (Jpn Circ J 2001; 65: 834-836)
\end{abstract}

Key Words: Coil embolization; Hemolysis; Patent ductus arteriosus

C oil embolization has been recently used for closure of patent ductus arteriosus (PDA), but because large PDA are difficult to close with this technique, it is generally used when the duct diameter is less than 3.5 $\mathrm{mm}$ ! However, there are some case reports in which large PDA were closed by placing more than 2 coils ${ }^{2,3}$ Serious complications of coil embolization are coil migration and hemolysis caused by a residual shunt, 4,5 the latter being reported as the major complication with approximately $1-2 \%$ occurrence, 6,7 Although a hemolytic complication usually needs additional coil embolization ${ }^{4,5}$ or surgical treatment, medical treatment may be preferable when the residual shunt is minimal and the degree of hemolysis is mild. We report 2 cases of large PDA that were treated by multiple coil embolization, complicated by hemolysis, but successfully managed by medical treatment.

\section{Case Reports}

Case 1

A 67-year-old woman was admitted for evaluation of dyspnea on exersion, paroxysmal atrial fibrillation (AF), and a continuous murmur of grade $4 / 6$ in the second left intercostal space. Physical examination revealed blood pressure of $120 / 60 \mathrm{mmHg}$ under medical treatment. The ECG finding was left ventricular hypertrophy with ST-T abnormality. Chest X-ray (CXR) showed moderate cardiomegaly

(Received September 4, 2000; revised manuscript received February 26, 2001; accepted March 1, 2001)

Departments of Internal Medicine and $*$ Pediatrics, Institute of Clinical Medicine, University of Tsukuba, Tsukuba-shi, Ibaraki-ken, Japan Mailing address: Kazuhiko Eda, MD, Cardiovascular Division, Department of Internal Medicine, Institute of Clinical Medicine, University of Tsukuba, 1-1-1 Ten-noudai, Tsukuba, Ibaraki 305-8575, Japan with increased pulmonary vascular markings. Color Doppler echocardiography showed a shunt flow in the pulmonary artery. Right heart catheterization revealed the pulmonary artery pressure was $30 / 14 \mathrm{mmHg}$ and there was an oxygen step-up at the level of the main pulmonary artery. The ratio of pulmonary to systemic flow was 2.0. Aortography revealed the shunt flow and a type $\mathrm{A} \mathrm{PDA}^{8}$ with a minimum ductal diameter of $5.3 \mathrm{~mm}$. Left ventriculography showed an increase in ventricular volume, but the ventricular wall motion and coronary angiogram were normal.

Because the ductal diameter was relatively large to be closed by embolization with one coil, we planned to deliver multiple Jackson detachable coils (MWCE-8-PDA5; diameter $8 \mathrm{~mm}$ and 5 loops, Cook Cardiology, Bjaeverskov, Denmark). Two 7Fr catheters were inserted into the femoral vein and advanced from the pulmonary artery through the

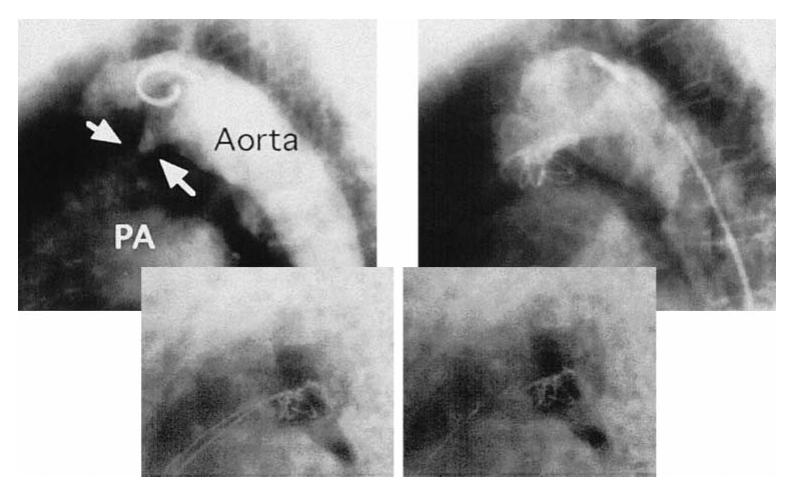

Fig 1. Case 1. Imaging before the treatment (Left upper). Delivery of the 2 coils, before they had detached (Left lower). The 2 coils after they had detached (Right lower). Imaging after the treatment (Right upper). 
Table 1 Postoperative Progress in Case 1

\begin{tabular}{|c|c|c|c|c|c|c|c|}
\hline & \multicolumn{7}{|c|}{$P O D$} \\
\hline & 0 & 1 & 2 & 3 & 4 & 5 & 6 \\
\hline Murmur & $I I / V I$ & $I I / V I$ & $I / V I$ & $I / V I$ & $I / V I$ & $I / V I$ & $I / V I$ \\
\hline Macrohematuria & +++ & ++ & ++ & - & - & - & \\
\hline Microhematuria & & +++ & & & +++ & & + \\
\hline Jaundice & & ++ & ++ & + & + & - & - \\
\hline Hemoglobin $(\mathrm{g} / \mathrm{dl})$ & 13.7 & 12.1 & & & 10.1 & & 9.3 \\
\hline GOT (IU/L) & 24 & 662 & & & 130 & & 35 \\
\hline$L D H(I U / d l)$ & 526 & 3,211 & & & 4,266 & & 2,160 \\
\hline Total bilirubin $(\mathrm{mg} / \mathrm{dl})$ & 1.0 & 5.1 & & & 1.6 & & 0.9 \\
\hline$B U N(m g / d l)$ & 19.1 & 8.7 & & & 10.8 & & 10.7 \\
\hline $\mathrm{Cr}(\mathrm{mg} / \mathrm{dl})$ & 0.6 & 0.6 & & & 0.9 & & 0.9 \\
\hline Haptoglobin (U/day) & & 8,000 & & & 8,000 & 6,000 & \\
\hline Antiplasmin (mg/day) & & & & & 2,000 & & \\
\hline
\end{tabular}

POD, postoperative date; GOT, glutamic oxaloacetic transaminase (normal range, 8-40IU/L); LDH, lactate dehydrogenase (normal range, 300-600 IU/dl); total bilirubin (normal range, 0.5-1.1 mg/dl); BUN, blood urea nitrogen (normal range, 8-20 mg/dl); $\mathrm{Cr}$, creatinine (normal range, $0.4-1.1 \mathrm{mg} / \mathrm{dl}$ ).

PDA to the descending aorta. First, we installed mandrill guide wires in the ampulla membranaceae and let the 2 coils slowly drop out. We then entangled the coils, pulled them back and fixed them in the PDA. Finally, we detached the coils from the catheters (Fig 1). After the coil embolization the calculated pulmonary-to-systemic flow ratio was normalized to 1.0 and color Doppler showed a trivial shunt flow. Nevertheless, aortography showed a slight residual shunt flow and a systolic murmur of grade $2 / 6$ was still audible. We tried to place one more coil from arterial side, and then from venous side, but it was impossible because the guide wire could not pass. Therefore we finished the procedure, hoping that the residual shunt would be closed by clot emphraxis. On echocardiography immediately after the operation, the residual shunt hardly could be detected.

Several hours after the procedure, the patient's urine began darken and urinalysis revealed hemoglobinuria. Blood analysis revealed elevated lactate dehydrogenase (4,266 IU/dl; normal range, 300-600) and total serum bilirubin $(5.1 \mathrm{mg} / \mathrm{dl}$; normal range, $0.5-1.1)$ and a fall in hemoglobin from 13.7 to $9.3 \mathrm{~g} / \mathrm{dl}$, all indicative of a hemolytic complication from the residual PDA shunt. To protect the kidney from hemoglobinuria, we administered fluids and haptoglobin by drip infusion $(6,000-10,000$ $\mathrm{U} /$ day), using hemoglobinuria as the marker of hemolysis. In addition, we continuously infused antiplasmin $(2,000$ $\mathrm{mg}$ /day) to promote clot emphraxis in the PDA and lowered the blood pressure with a calcium blocker (nicardipine) to decrease the shunt flow. Consequently, the murmur decreased to grade 1/6 and hemolysis disappeared 6 days after the procedure (Table 1). Three months after the coil embolization, the murmur was not audible, and neither urinalysis nor blood chemistry indicated hemolysis. The cardiothoracic ratio on CXR had reduced from $58 \%$ to $50 \%$ and the patient was not experiencing dyspnea on exertion or paroxysmal AF.

\section{Case 2}

A 71-year-old woman was referred for evaluation of congestive heart failure. For the 6 months prior to admission, she had began to suffer from dyspnea on exertion and edema of both legs. Physical examination revealed blood pressure of $136 / 88 \mathrm{mmHg}$ under medical treatment and a
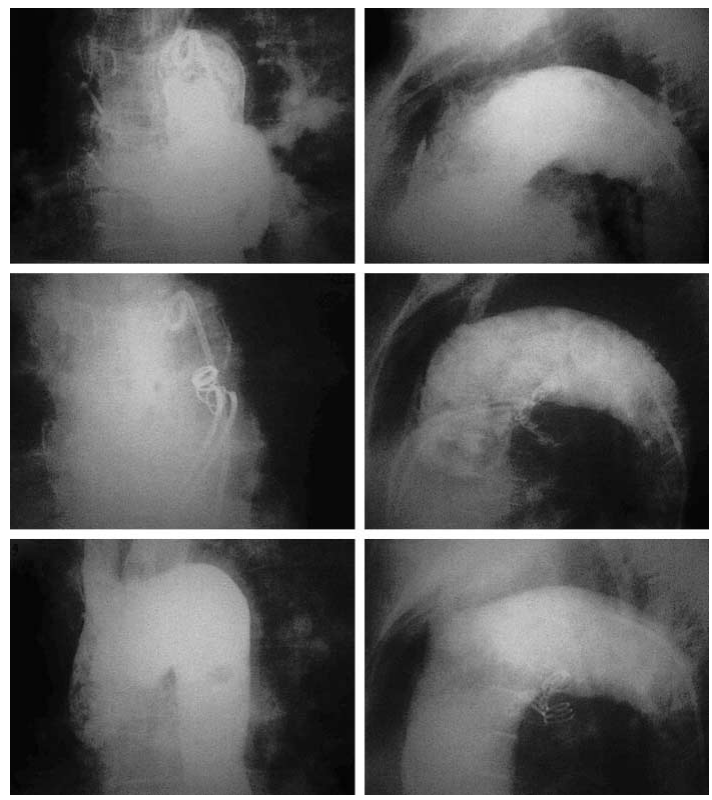

Fig 2. Case 2. (Left) AP view, (Right) lateral view. (Upper) Preoperative, (Middle) peri-operative, (Lower) postoperative.

grade $3 / 6$ continuous murmur in the left infra-clavicular area radiating posteriorly. The ECG findings were AF and high voltage in the left precordial leads with ST depression. CXR showed cardiomegaly with a cardiothracic ratio of $59 \%$. Echocardiography confirmed the presence of a PDA and showed a dilated left atrium and ventricle. Cardiac catheterization showed an increased pressure of 44/10 $\mathrm{mmHg}$ and an oxygen step-up in the main pulmonary artery. The calculated pulmonary-to-systemic flow ratio was 2.3 . Aortography showed a type A PDA with a minimum ductal diameter of $5.5 \mathrm{~mm}$ and moderate left to right shunt flow.

In order to close the PDA, the same procedure and system as used in case 1 was followed and coil embolization with 2 coils (MWCE-8-PDA5; diameter $8 \mathrm{~mm}$ and 5 loops, Cook Cardiology) was carried out. Although the oxygen content analysis showed the calculated pulmonaryto-systemic flow ratio had normalized to 1.0 , a systolic 
murmur of grade $2 / 6$ was still audible after the embolization. We tried to place one more coil into PDA, but again it was impossible to pass the guide wire. Aortography showed only slight residual shunt flow (Fig 2). On the postoperative echocardiography, the residual shunt could barely be detected. Several hours later gross hematuria occurred; hemoglobin fell from 12.7 to $9.9 \mathrm{~g} / \mathrm{dl}$ and there were increased levels of glutamic oxaloacetic transaminase (51 $\mathrm{IU} / \mathrm{L})$, lactate dehydrogenase $(1,350 \mathrm{IU} / \mathrm{dl})$ and total serum bilirubin $(1.2 \mathrm{mg} / \mathrm{dl})$. To prevent a hemolytic complication we administered haptoglobin by drip infusion $(4,000$ U/day) and continuously infused antiplasmin (1,000-2,000 $\mathrm{mg}$ /day). Hemolysis disappeared 7 days after the procedure and the patient was discharged.

\section{Discussion}

In 1967 the Porstmann device was first reported as a catheter therapy for PDA? Then the disk occluder of Rashkind and Cuaso was reported in 197910 and in 1991 a buttoned device was used!1 However, all these devices required relatively large sheaths and so were not suitable for young or small patients. In 1992, coil embolization was reported as an alternative procedure ${ }^{12}$ was further developed with detachable coils in 1994 and has now become a popular choice for repair of PDA. However, coil embolization is difficult in a large arterial canal, and is more likely to be associated with some complications, especially when the ductal diameter is more than $3.5 \mathrm{~mm}$.

There have been reports of the use of more than 2 coils for large PDA $2,3,6,7,13,14$ and we also performed coil embolization in 2 adult cases of PDA in which ductal diameter was more than $5 \mathrm{~mm}$. We simultaneously detached and placed 2 coils in the PDA and succeeded in closing it functionally. However, dropout of coils, arctation of the left pulmonary artery and hemolysis are complications of coil embolization, with hemolysis being the major complication, occurring in approximately $1-2 \%$ of cases $2,6,7$

If the hemolysis progresses, hemoglobinuria induces renal damage and progression of anemia aggravates the congestive heart failure, so it must prevented. The mechanism for the hemolysis is considered to be mechanical stress through the residual shunt, which destroys the blood cells intravascularly. The magnitude of the hemolysis may depend on the degree of arctation, velocity of the shunt flow, platelet function, and the activity of fibrinolysis factors. In a large PDA, there is a greater possibility that the ductal shunt flow will remain even when multiple embolization coils are used and if this occurs, the placement of additional coils is recommended $4,5,14,15$ If the additional coils cannot be inserted, the arterial canal must be closed surgically? Retrieval of the embolized coils through a catheter is another option, but is not recommended when multiple coils are implanted because of the high risk of migration of the coils.

In the present cases, we tried to embolize completely by adding another coil, but it was impossible. However, because the residual shunt was minimal and the anemia was mild, we elected to manage it medically with haptoglobin and antiplasmin infusion, together with fluids and lowering of the blood pressure to reduce the shunt flow. The degree of hemoglobinuria and level of serum bilirubin, as well as renal function, are the markers of the effectiveness of the treatment. However, if the hemolysis appears to be progress after a few days, surgical treatment may be required.

In conclusion, coil embolization may be carried out even in a relatively large PDA, by placing several coils simultaneously. The major complication of the coil embolization is hemolysis, which occurs because of residual shunt flow. A second procedure of additional coil embolization or surgical repair is usually considered, but the hemolytic complication may be curable by medication when the residual shunt is trivial. The upper limit of the diameter of the PDA that is amenable to coil embolization remains to be investigated.

\section{References}

1. Syamasundar PR: Transcatheter occlusion of patent ductus arteriosus: Which method to use and which ductus to close. Am Heart J 1996; 132: $905-909$

2. Celiker A, Qureshi SA, Bilgic A, Carminati M, Kirk R, Tynan M, et al: Transcatheter closure of patent arterial ducts using controlledrelease coils. Eur Heart J 1997; 18: 450-454

3. Nishimoto K, Ino T, Ohkubo M, Akimoto K, Yabuta K: Mid-term follow-up results of coil embolization for patent ductus arteriosus. $J$ Cardiol 1997; 30: $131-136$

4. Shim D, Wechsler DS, Lloyd TR, Beekman RH: Hemolysis following coil embolization of a patent ductus arteriosus. Cathet Cardiovasc Diagn 1996; 39: 287-290

5. Henry G, Danilowicz D, Verma R: Severe hemolysis following partial coil-occlusion of patent ductus arteriosus. Cathet Cardiovasc Diagn 1996; 39: 410-412

6. Tomita H, Fuse S, Akagi T, Matsumoto Y, Murakami Y, Chiba S, et al: Hemolysis complicating coil occlusion of patent ductus arteriosus. Cathet Cardiovasc Diagn 1998; 43: 50-53

7. Jacob JLB, Coelho WMC, Machado NCS, Garzon SAC: Transcatheter occlusion of patent ductus arteriosus using coil embolization. Int J Cardiol 1997; 60: $133-138$

8. Krichenko A, Benson LN, Burrows P, Moes CAF, McLaughlin P, Freedom RM, et al: Angiographic classification of the isolated, persistently patent ductus arteriosus and implications for percutaneous catheter occlusion. Am J Cardiol 1989; 1: 877-880

9. Porstmann W, Wierny L: Der Verschluss des Ductus arteriosus persistens ohne Thorakotomie. Thoraxchirurgie 1967; 15: 109-203

10. Rashkind WJ, Cuaso CC: Transcatheter closure of a patent ductus arteriosus: Successful use in a $3.5 \mathrm{~kg}$ infant. Pediatr Cardiol 1979; 1: 3-7

11. Rao PS, Wilson AD: Transcatheter closure of patent ductus arteriosus with buttoned device: First successful clinical application in a child. Am Heart J 1991; 121: 1799-1802

12. Cambier PA, Kirby WC, Wortham DC, Moore JW: Percutaneous closure of small $(<2.5 \mathrm{~mm})$ patent ductus arteriosus using coil embolization. Am J Cardiol 1992; 69: 815-816

13. Podnar T, Masura J: Percutaneous closure of patent ductus arteriosus using special screwing detachable coil. Cathet Cardiovasc Diagn 1997; 41: 386-391

14. Oishi Y, Okamoto M, Sueda T, Hashimoto M, Karakawa S, Akita T: Transcatheter coil embolization of large-size patent ductus arteriosus in adult patients: Usefulness and problems. Jpn Circ J 1999; 63: 994-998

15. Tomita H, Fuse S, Akagi T, Koike K, Kamada M, Kamiya T, et al: Coil occlusion for patent ductus arteriosus in Japan. Jpn Circ J 1997; 61: $997-1003$ 